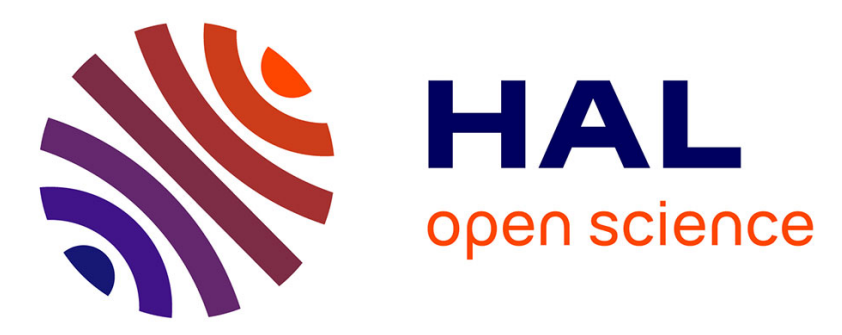

\title{
A second gradient material resulting from the homogenization of an heterogeneous linear elastic medium
}

Catherine Pideri, Pierre Seppecher

\section{- To cite this version:}

Catherine Pideri, Pierre Seppecher. A second gradient material resulting from the homogenization of an heterogeneous linear elastic medium. Continuum Mechanics and Thermodynamics, 1997, 9 (5), pp.241-257. hal-00527291

\section{HAL Id: hal-00527291 \\ https://hal.science/hal-00527291}

Submitted on 18 Oct 2010

HAL is a multi-disciplinary open access archive for the deposit and dissemination of scientific research documents, whether they are published or not. The documents may come from teaching and research institutions in France or abroad, or from public or private research centers.
L'archive ouverte pluridisciplinaire HAL, est destinée au dépôt et à la diffusion de documents scientifiques de niveau recherche, publiés ou non, émanant des établissements d'enseignement et de recherche français ou étrangers, des laboratoires publics ou privés. 


\title{
A second gradient material resulting from the homogenization of an heterogeneous linear elastic medium
}

\author{
C. Pideri and P. Seppecher
}

Laboratoire d'Analyse Non Linéaire Appliquée, Université de Toulon et du Var, BP 132 - 83957 La Garde Cedex, France

\begin{abstract}
Homogenization may change fundamentally the constitutive laws of materials. We show how a heterogeneous Cauchy continuum may lead to a non Cauchy continuum. We study the effective properties of a linear elastic medium reinforced periodically with thin parallel fibers made up of a much stronger linear elastic medium and we prove that, when the Lamé coefficients in the fibers and the radius of the fibers have appropriate order of magnitude, the effective material is a second gradient material, i.e. a material whose energy depends on the second gradient of the displacement.
\end{abstract}

\section{Introduction}

Continuum mechanics is usually understood as a homogenized description of materials which are heterogeneous at the microscopic level. Then, it is natural to expect from any general theory of continuum mechanics to be stable by homogenization procedures. We prove in this paper that the class of Cauchy continua does not enjoy this stability property. Indeed, we show that the effective properties of some periodic elastic material have to be described by a second gradient theory.

We consider a composite material made up of an elastic matrix reinforced with elastic fibers. Both materials are isotropic linear elastic materials, the Lamé coefficients in the fibers being larger than in the matrix. The structure is periodic: we assume that the fibers are parallel cylinders with the circular section arranged along a square lattice (see Fig. 1).

Homogenization procedure consists in studying the limit behaviour of the material when the period of the structure tends to zero. What is the behaviour of the other physical quantities as the period tends to zero? The effective properties of the material strongly depend on them: when the elasticity coefficients in the fibers are of the same order of magnitude as in the matrix and when the radius of the fibers is of the same order of magnitude as the period, the problem is a classic one in homogenization theory: the effective material is still a linear elastic material whose coefficients can be expressed in terms of the geometry and of the elasticity coefficients of the matrix and the fibers [18]. We study a different case: we want to describe a composite medium reinforced by very thin and very rigid fibers. Then, it is natural to assume that the radius of the fibers tends to zero faster than the period and that the elasticity coefficients in the fibers tend to infinity.

Let us now fix some notations: by convention, we choose the characteristic length of the domain as the unit length. The period of the lattice is denoted by $\varepsilon$. We study the limit $\varepsilon \rightarrow 0$ and every quantity which is not assumed to be constant as $\varepsilon$ tends to zero, is indiced by $\varepsilon$. For instance, the radius of the fibers is denoted by $r_{\varepsilon}$, the Lamé coefficients in the fibers are denoted by $\lambda_{\varepsilon}$ and $\mu_{\varepsilon}$ while the Lamé coefficients in the matrix are denoted by $\lambda_{0}$ and $\mu_{0}$. Then our assumptions read

$$
\frac{r_{\varepsilon}}{\varepsilon} \rightarrow 0 \quad, \quad \lambda_{\varepsilon} \rightarrow \infty \quad, \quad \mu_{\varepsilon} \rightarrow \infty
$$


This situation has already been studied by D. Caillerie [7] who, setting $\lambda_{\varepsilon}=\left(r_{\varepsilon} / \varepsilon\right)^{-\theta}, \mu_{\varepsilon}=\left(r_{\varepsilon} / \varepsilon\right)^{-\theta}$, considered in two cases the limit $\left(\varepsilon, r_{\varepsilon} / \varepsilon\right) \rightarrow(0,0):\left(r_{\varepsilon} / \varepsilon \rightarrow 0\right.$ then $\left.\varepsilon \rightarrow 0\right)$ and $\left(\varepsilon \rightarrow 0\right.$ then $\left.r_{\varepsilon} / \varepsilon \rightarrow 0\right)$. He found that both cases lead to an elastic material but that the homogenized elasticity coefficients depend on the limit procedure: the two limits $\varepsilon \rightarrow 0$ and $\left(r_{\varepsilon} / \varepsilon\right) \rightarrow 0$ do not commute. Here we let $\frac{r_{\varepsilon}}{\varepsilon}, \mu_{\varepsilon}^{-1}$ and $\lambda_{\varepsilon}^{-1}$ tend to zero together and assume that:

$$
\lim _{\varepsilon \longrightarrow 0} \frac{r_{\varepsilon}}{\varepsilon}=0, \quad \lim _{\varepsilon \longrightarrow 0} \varepsilon^{2} \log \left(r_{\varepsilon}\right)=0, \quad \lim _{\varepsilon \longrightarrow 0} \frac{\mu_{\varepsilon} r_{\varepsilon}^{4}}{\varepsilon^{2}}=\mu_{1}>0, \quad \lim _{\varepsilon \longrightarrow 0} \frac{\lambda_{\varepsilon}}{\mu_{\varepsilon}}=\ell .
$$

This particular scaling leads to a very different limit behaviour. We prove that the energy of the effective material depends not only on the strain tensor (as a classical elastic material) but also on the second gradient of the displacement. This result has been announced in [17].

Materials whose energy depends on the second gradient of the displacement cannot be considered as Cauchy continua otherwise one would be led to a thermodynamic paradox [12]. This paradox can be removed by extending the thermodynamical framework [12] but the fundamental point is that the Cauchy stress tensor is not sufficient to describe internal forces [20]. External forces concentrate along any edge of the boundary and the Cauchy theorem defining the Cauchy stress tensor cannot be applied $[10,11]$. Moreover, a supplementary boundary condition is needed to write well-posed problems, which is unusual and not intuitive [19]. The simplest way to describe these media is to use the second gradient theory [13, 14] or to consider them as Cosserat media [8]. Our result gives a new example of such a material together with a "microscopic" interpretation of its special features.

We emphasize that, going to the limit, the differential order of the energy changes (as does the system of partial differential equations associated with equilibrium). Such a change is not usual in homogenization theory. It arises in rod or plate theories [1] but seems then to be connected with a change of dimension. Our result shows that this is not necessary. Notice also that such a change in the differential order of the energy can not arise when considering scalar problems (like thermal conductivity problems). Indeed, consider a sequence of energies which are quadratic functions of the gradient of a scalar quantity $u$; these energies decrease when truncating $u$ and this property is preserved when going to the limit. Then, a representation theorem for Dirichlet forms [6,5] assures that the limit energy can be represented as the sum of a term depending on $u$ and $\nabla u$ and a non-local term of the form: $\iint_{\Omega \times \Omega} K(x, y)(u(x)-u(y))^{2} d x d y$. In other words, we can expect non-local effects but no increase of the differential order. Our result shows that this argument cannot be extended to elasticity problems.

Non-local effects actually arise for some scalar singular perturbation problems [5, 4] and we should probably have obtained non-local effects if assuming that $\varepsilon^{2}\left|\log \left(r_{\varepsilon}\right)\right|$ converges to a finite positive value instead of zero. We do not have non-local effects under our assumptions: the second gradient part of the limit energy cannot be interpreted, as it is often done, as the limit of non-local interactions whose range is very short.

Our study is variational. We identify the $\Gamma$-limit $E_{0}$ of the energy $E_{\varepsilon}$ of our composite material. The notion of $\Gamma$-convergence corresponds to the intuitive notion of convergence of models: the result is obtained without considering external forces, it remains valid in presence of body forces (for definition and properties of $\Gamma$-convergence, refer to [9]).

The limit energy is made explicit in Sect. 2 where we state precisely our result. Section 3 is devoted to the more difficult part of the proof: considering a sequence of displacement fields $\left(u_{\varepsilon}\right)$ converging to some $u$, we have to express the lower bound for the energy $E_{\varepsilon}\left(u_{\varepsilon}\right)$ in terms of $u$. This needs an accurate description of the asymptotic behaviour of $u_{\varepsilon}$. Especially $u_{\varepsilon}$ has to be described at the scale $r_{\varepsilon}$ inside the fibers: we need a multiscale notion of convergence. However, we do not expect any periodicity with period $r_{\varepsilon}$; the classical notions of multiscale convergence (as defined in [16] or [2]) are not convenient. In Subsect. 3.1, we develop an adapted notion of double-scale convergence which describes the asymptotic behaviour of $u_{\varepsilon}$ in the fibers, that is in a set of scale $r_{\varepsilon}$ but with periodicity $\varepsilon$. Section 4 is devoted to the end the proof: for any admissible displacement field $u$ we have to construct an approximating sequence $u_{\varepsilon}$ whose limit energy is not larger than $E_{0}(u)$. Such an approximation is obtained by choosing $u_{\varepsilon}=u$ in the main part of the matrix, a rod-like displacement field in the fibers and a suitable interpolation in transition layers around each fiber. 


\section{The main result}

\subsection{Notations and Geometry}

In $\mathbb{R}^{3}$ we refer to a point $x$ by its Cartesian coordinates $\left(x_{1}, x_{2}, x_{3}\right)$. In the same way the coordinates of any vector $u$ are denoted by $\left(u_{1}, u_{2}, u_{3}\right)$. The symmetric part of the gradient of $u$ (the strain tensor) is denoted by $e(u):=\left(\nabla u+\nabla u^{t}\right) / 2$. This tensor belongs to the set of 3-3 symmetric matrices which we denote by $\mathscr{M}$. The trace of a matrix $A$ is denoted by $\operatorname{Tr}(A)$.

We use the summation convention, but, as we consider two and three dimensional spaces, we adopt the following convention: a repeated Latin index is summed from 1 to 3 while a Greek index is summed from 1 to 2 .

For every Borel set $D$ and $u \in \mathrm{L}^{1}(D)$, we denote by $|D|$ the Lebesgue measure of $D$ and by $f_{D} u d x$ the mean value of $u$ on $D: f_{D} u d x:=|D|^{-1} \int_{D} u d x$.

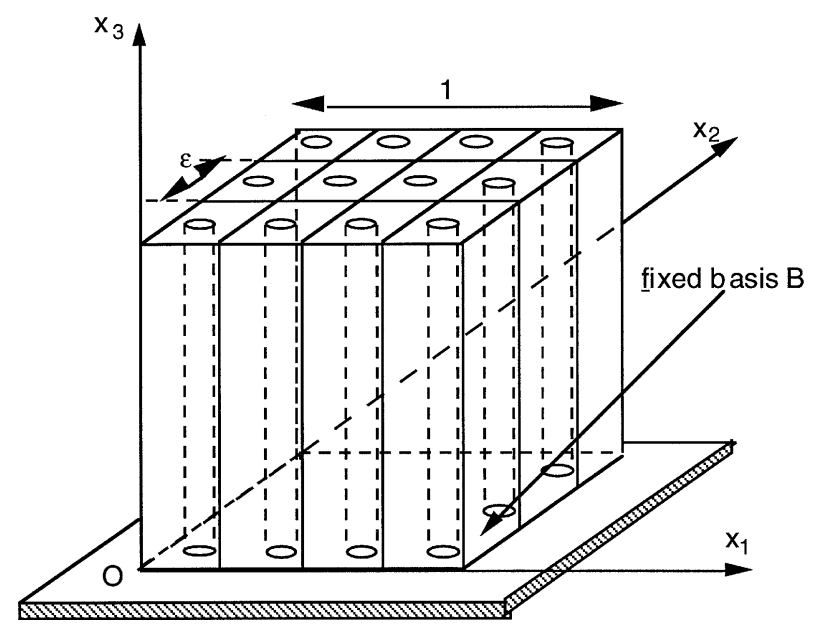

Fig. 1. The composite material, $\Omega$

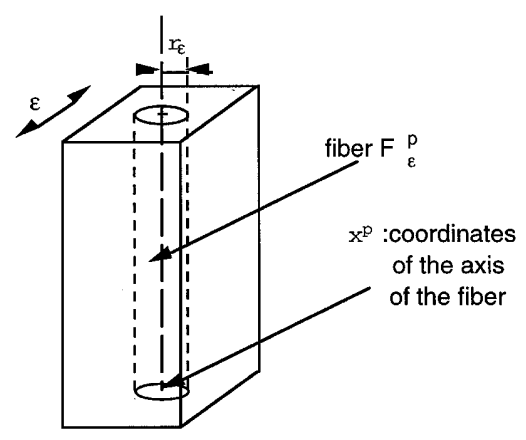

Fig. 2. A period $P_{\varepsilon}^{p}$ of the composite material

In order to describe the periodic structure of our composite material, we introduce two positive real parameters $\varepsilon$ and $r_{\varepsilon}\left(r_{\varepsilon} \leq \varepsilon\right)$. Then we define the projection $p_{\varepsilon}$ :

$$
\begin{array}{ll}
p_{\varepsilon}: \quad & \mathbb{R}^{2} \longrightarrow \mathbb{R}^{2} \\
& \left(x_{1}, x_{2}\right) \longmapsto\left(\varepsilon\left(\mathrm{E}\left(\frac{x_{1}}{\varepsilon}\right)+\frac{1}{2}\right), \varepsilon\left(\mathrm{E}\left(\frac{x_{2}}{\varepsilon}\right)+\frac{1}{2}\right)\right),
\end{array}
$$

where $\mathrm{E}(t)$ denotes the integer part of a real $t$ and we define the periodic function $y^{\varepsilon}$ by

$$
\begin{array}{ll}
y_{\varepsilon}: \quad \mathbb{R}^{3} \longrightarrow \mathbb{R}^{2} \\
\quad\left(x_{1}, x_{2}, x_{3}\right) \longmapsto r_{\varepsilon}^{-1}\left[\left(x_{1}, x_{2}\right)-p_{\varepsilon}\left(x_{1}, x_{2}\right)\right] .
\end{array}
$$


Next, we define the sets $F_{\varepsilon}$ and $M_{\varepsilon}$, referred to as "the fibers" and "the matrix" respectively, by:

$$
F_{\varepsilon}:=\left\{x \in \mathbb{R}^{3}:\left|y_{\varepsilon}(x)\right|<1\right\} \quad, \quad M_{\varepsilon}:=\mathbb{R}^{3} \backslash F_{\varepsilon}
$$

We assume that the composite material lies in the cube $\Omega=(0,1)^{3}$ and we denote by $\mathscr{B}$ its "lower" face: $\mathscr{B}=(0,1)^{2} \times\{0\}$. On $\Omega$ the projection $p_{\varepsilon}$ ranges onto a finite set of points which we denote by $\left\{x^{p} ; p \in \mathscr{P}_{\varepsilon}:=\left\{1 \ldots \frac{1}{\varepsilon^{2}}\right\}\right\}$. The domain $\Omega$ is the union of the $\varepsilon^{-2}$ parallelepipeds $P_{\varepsilon}^{p}:=\left\{x \in \Omega: p_{\varepsilon}(x)=\right.$ $\left.x^{p}\right\}$ which correspond to the periods of $y_{\varepsilon}$.

The fiber contained in the period $P_{\varepsilon}^{p}$ (a circular cylinder of radius $r_{\varepsilon}$, see Fig. 2) is denoted by $F_{\varepsilon}^{p}:=F_{\varepsilon} \cap P_{\varepsilon}^{p}$. The total volume of the fibers contained in $\Omega$ is $\left|F_{\varepsilon} \cap \Omega\right|=\sum_{p \in \cap P_{\varepsilon}}\left|F_{\varepsilon}^{p}\right|=\pi r_{\varepsilon}^{2} \varepsilon^{-2}$.

\subsection{Elastic energy}

We assume that $F_{\varepsilon}$ and $M_{\varepsilon}$ consist of two different isotropic elastic materials: we define for every Borel set $D$ the matrix energy by

$$
E^{m}(D, u):=\int_{D}\left[\frac{\lambda_{0}}{2}(\operatorname{Tr}(e(u)))^{2}+\mu_{0} e(u)^{2}\right] d x,
$$

where $\left(\lambda_{0}, \mu_{0}\right)$ denote the (positive) elasticity Lamé coefficients in the matrix. In the same way, we define the fiber energy by

$$
E_{\varepsilon}^{f}(D, u):=\int_{D}\left[\frac{\lambda_{\varepsilon}}{2}(\operatorname{Tr}(e(u)))^{2}+\mu_{\varepsilon} e(u)^{2}\right] d x,
$$

where $\left(\lambda_{\varepsilon}, \mu_{\varepsilon}\right)$ denote the (positive) elasticity Lamé coefficients in the fibers.

We assume perfect adhesion between the matrix and the fibers. Moreover, we assume that both materials are fixed to the plane $\left\{x_{3}=0\right\}$. Then, for any displacement $u \in \mathrm{L}^{2}\left(\Omega, \mathbb{R}^{3}\right)$, we define the total energy $E_{\varepsilon}(u):=E_{\varepsilon}(\Omega, u)$ of our composite material by

$$
E_{\varepsilon}(\Omega, u):= \begin{cases}E^{m}\left(M_{\varepsilon} \cap \Omega, u\right)+E_{\varepsilon}^{f}\left(F_{\varepsilon} \cap \Omega, u\right), & \text { if } u \in \mathrm{H}^{1}\left(\Omega, \mathbb{R}^{3}\right) \\ +\infty & \text { and } u=0 \text { on } \mathscr{B} \\ & \text { otherwise }\end{cases}
$$

\subsection{Order of magnitude of the different parameters}

In order to study the $\Gamma$-limit of $E_{\varepsilon}$ as $\varepsilon$ tends to zero, we must specify our assumptions upon the behaviour of $r_{\varepsilon}, \lambda_{\varepsilon}$ and $\mu_{\varepsilon}$ as $\varepsilon$ tends to zero: we assume that $r_{\varepsilon}$ obeys the limit relations

$$
\begin{aligned}
& \lim _{\varepsilon \longrightarrow 0} \frac{r_{\varepsilon}}{\varepsilon}=0, \\
& \lim _{\varepsilon \longrightarrow 0} \varepsilon^{2} \log \left(r_{\varepsilon}\right)=0
\end{aligned}
$$

and that $\mu_{\varepsilon}$ and $\lambda_{\varepsilon}$ fulfill the limit conditions

$$
\begin{aligned}
& \left.\lim _{\varepsilon \longrightarrow 0} \frac{\mu_{\varepsilon} r_{\varepsilon}^{4}}{\varepsilon^{2}}=\mu_{1} \in\right] 0,+\infty[ \\
& \lim _{\varepsilon \rightarrow 0} \frac{\lambda_{\varepsilon}}{\mu_{\varepsilon}}=\ell \in[0,+\infty[
\end{aligned}
$$

Assumption (5) states that the fibers are much thinner than the period of the medium; it is one of our basic assumptions. However, they cannot be too thin, otherwise the connection between the displacement fields in the matrix and in the fibers disappears when $\varepsilon$ tends to zero. This fact can be explained as follows: if the radius of the fibers is infinitely smaller than $\varepsilon$, the fibers behave like one-dimensional media and it is well 
known that a one-dimensional medium has no connection with a three-dimensional elastic medium. We will see later that restriction (6) assures that the global displacement of each fiber coincides with the displacement of the matrix when $\varepsilon$ tends to zero. Note that assumption (6) is not very restrictive: any power law $r_{\varepsilon}=\varepsilon^{\gamma}$, $\gamma>1$ is admissible.

The energy of a bent rod is related to its curvature, that is to the second gradient of its displacement. As our goal is to obtain second gradient effects, we expect each fiber to behave like a rod. The bending stiffness of a unique fiber is $\frac{\pi}{4} r_{\varepsilon}^{4} \mu_{\varepsilon} \frac{3 \lambda_{\varepsilon}+2 \mu_{\varepsilon}}{\lambda_{\varepsilon}+\mu_{\varepsilon}}$ (refer to any textbook for mechanics of structures or to the pioneering work of St. Venant [3]). Assumptions (7) and (8) state that this stiffness is of the order of $\varepsilon^{2}$, the inverse of the number $\left(\varepsilon^{-2}\right)$ of fibers.

\subsection{The main result}

Our result states that $E_{\varepsilon} \Gamma$-converges in $\mathrm{L}^{2}\left(\Omega, \mathbb{R}^{3}\right)$ to $E_{0}$ defined by:

$$
E_{0}(u)=\left\{\begin{aligned}
E^{m}(\Omega, u)+ & \int_{\Omega} \frac{k}{2}\left[\left(\frac{\partial^{2} u_{1}}{\partial x_{3}^{2}}\right)^{2}+\left(\frac{\partial^{2} u_{2}}{\partial x_{3}^{2}}\right)^{2}\right] d x \\
& \text { if } u \in \mathrm{H}^{1}\left(\Omega, \mathbb{R}^{3}\right), \frac{\partial^{2} u}{\partial x_{3}^{2}} \in \mathrm{L}^{2}\left(\Omega, \mathbb{R}^{3}\right), \\
& u_{3}=0 \text { a.e. in } \Omega, u=\frac{\partial u}{\partial x_{3}}=0 \text { a.e. on } \mathscr{B}, \\
+\infty & \text { otherwise. }
\end{aligned}\right.
$$

where

$$
k=\frac{\pi 3 \ell+2}{4 \ell+1} \mu_{1}
$$

More precisely we have the following:

Theorem 1. i) Let $u_{\varepsilon}$ be a sequence such that $E_{\varepsilon}\left(u_{\varepsilon}\right)$ is bounded. Then $u_{\varepsilon}$ is strongly relatively compact in $\mathrm{L}^{2}\left(\Omega, \mathbb{R}^{3}\right)$.

ii) Moreover, for any sequence $u_{\varepsilon}$ converging to $u$ in $\mathrm{L}^{2}\left(\Omega, \mathbb{R}^{3}\right)$, the following lower bound inequality holds:

$$
\liminf _{\varepsilon \longrightarrow 0} E_{\varepsilon}\left(u_{\varepsilon}\right) \geq E_{0}(u)
$$

iii) Conversely, for every $u$ in $\mathrm{L}^{2}\left(\Omega, \mathbb{R}^{3}\right)$, there exists an approximating sequence $u_{\varepsilon}$ in $\mathrm{L}^{2}\left(\Omega, \mathbb{R}^{3}\right)$ such that

$$
u_{\varepsilon} \longrightarrow u \operatorname{in} \mathrm{L}^{2}\left(\Omega, \mathbb{R}^{3}\right), \quad \underset{\varepsilon \longrightarrow 0}{\limsup } E_{\varepsilon}\left(u_{\varepsilon}\right) \leq E_{0}(u) .
$$

Proof of assertion (i): It is clear from assumptions (5), (7) and (8) that $\lambda_{\varepsilon}$ and $\mu_{\varepsilon}$ tend to infinity. Then there exists a positive real $c$ such that $E_{\varepsilon}(u) \geq c \int_{\Omega} e(u)^{2} d x$ for every $u$ in $\mathrm{H}^{1}\left(\Omega, \mathbb{R}^{3}\right)$. Due to Korn's inequality, there exists a positive real $C$ such that $E_{\varepsilon}(u) \geq C\|u\|_{\mathrm{H}^{1}\left(\Omega, \mathbb{R}^{3}\right)}$. The sequence $u_{\varepsilon}$ is then bounded in $\mathrm{H}^{1}\left(\Omega, \mathbb{R}^{3}\right)$ : it is strongly relatively compact in $\mathrm{L}^{2}\left(\Omega, \mathbb{R}^{3}\right)$.

The proofs of (ii) and (iii) are less straightforward. They are given in the following two sections.

\section{Proof of the lower bound inequality}

\subsection{Preliminaries, double-scale convergence}

Let us denote by $D_{1}$ the unit disk of $\mathbb{R}^{2}$ and by $\mathscr{D}$ the set of functions $\mathscr{D}:=C_{c}^{\infty}\left(\Omega \times D_{1}, \mathbb{R}\right)$. We associate to the sequence of sets $\left(F_{\varepsilon} \cap \Omega\right)$ the following "double scale" convergence:

Definition: We say that a sequence $u_{\varepsilon}$ in $\mathrm{L}^{2}(\Omega, \mathbb{R})$ double scale converges to $v \in \mathrm{L}^{2}\left(\Omega \times D_{1}, \mathbb{R}\right)$ and we write $u_{\varepsilon} \longrightarrow v$ if and only if 


$$
\forall \varphi \in \mathscr{D}, \quad f_{F_{\varepsilon} \cap \Omega} u_{\varepsilon}(x) \varphi\left(x, y_{\varepsilon}(x)\right) d x \rightarrow f_{\Omega} f_{D_{1}} v(x, y) \varphi(x, y) d y d x
$$

This definition is extended to vector field or tensor field sequences: we say that such sequences d.s.-converge if and only if every component is d.s.-convergent.

Remark 1. For every function $\Phi \in \mathscr{D}$,

$$
u_{\varepsilon} \longrightarrow v \Longrightarrow \Phi\left(., y_{\varepsilon}(.)\right) u_{\varepsilon}(.) \longrightarrow \Phi v
$$

Indeed, for every $\psi \in \mathscr{D}$, the product $\psi \Phi$ belongs to $\mathscr{D}$ and the result is obtained by applying the definition of the d.s.-convergence of $u_{\varepsilon}$ with $\varphi=\psi \Phi$.

Lemma 1. For every function $\Phi \in \mathscr{D}$ we have

$$
\Phi\left(., y_{\varepsilon}(.)\right) \longrightarrow \Phi
$$

Proof: For $\varphi \in \mathscr{D}$, let us compute the limit of $f_{F_{\varepsilon} \cap \Omega} \varphi\left(x, y_{\varepsilon}(x)\right) d x$. Using the Fubini theorem and changing variables in each fiber we get

$$
\begin{aligned}
& f_{F_{\varepsilon} \cap \Omega} \varphi\left(x, y_{\varepsilon}(x)\right) d x=\varepsilon^{2} \sum_{p \in \mathscr{P} \varepsilon} f_{F_{\varepsilon}^{p}} \varphi\left(x, y_{\varepsilon}(x)\right) d x \\
& \quad=\left|F_{\varepsilon} \cap \Omega\right|^{-1} r_{\varepsilon}^{2} \sum_{p \in \mathscr{P}_{\varepsilon}} \int_{0}^{1} \int_{D_{1}} \varphi\left(\left(x_{1}^{p}+r_{\varepsilon} y_{1}, x_{2}^{p}+r_{\varepsilon} y_{2}, x_{3}\right),\left(y_{1}, y_{2}\right)\right) d y d x_{3} .
\end{aligned}
$$

As the function $\varphi$ is uniformly continuous on $\Omega \times D_{1}$, we have the following uniform estimations:

$$
\left|\varphi\left(\left(x_{1}^{p}+r_{\varepsilon} y_{1}, x_{2}^{p}+r_{\varepsilon} y_{2}, x_{3}\right),\left(y_{1}, y_{2}\right)\right)-\varphi\left(\left(x_{1}^{p}, x_{2}^{p}, x_{3}\right),\left(y_{1}, y_{2}\right)\right)\right|=O\left(r_{\varepsilon}\right)
$$

and

$$
\left|\sum_{p \in \mathscr{P} \varepsilon} \varphi\left(\left(x_{1}^{p}, x_{2}^{p}, x_{3}\right), y\right) 1_{P_{\varepsilon}^{p}}(x)-\varphi(x, y)\right|=O(\varepsilon)
$$

which implies

$$
\left|\int_{0}^{1} \varepsilon^{2} \sum_{p \in \mathscr{P}_{\varepsilon}} \varphi\left(\left(x_{1}^{p}, x_{2}^{p}, x_{3}\right), y\right) d x_{3}-\int_{\Omega} \varphi(x, y) d x\right|=O(\varepsilon) .
$$

Hence

$$
\begin{aligned}
\lim _{\varepsilon \rightarrow 0} \int_{F_{\varepsilon} \cap \Omega} \varphi\left(x, y_{\varepsilon}(x)\right) d x & =\lim _{\varepsilon \rightarrow 0}\left|F_{\varepsilon} \cap \Omega\right|^{-1} r_{\varepsilon}^{2} \varepsilon^{-2} \int_{\Omega} \int_{D_{1}} \varphi(x, y) d y d x \\
& =f_{\Omega} f_{D_{1}} \varphi(x, y) d y d x .
\end{aligned}
$$

In other words, the constant function 1 d.s.-converges to itself. The lemma is proved by recalling Remark 1.

Lemma 2. Let $u_{\varepsilon}$ be a sequence in $\mathrm{L}^{2}(\Omega, \mathbb{R})$ such that $f_{F_{\varepsilon} \cap \Omega} u_{\varepsilon}^{2}(x) d x$ is bounded, then there exists a subsequence of $u_{\varepsilon}$ (still denoted by $u_{\varepsilon}$ ) and a function $v \in \mathrm{L}^{2}\left(\Omega \times D_{1}, \mathbb{R}\right)$ such that

$$
u_{\varepsilon} \rightarrow v
$$


Proof: Assume $f_{F_{\varepsilon} \cap \Omega} u_{\varepsilon}^{2}(x) d x \leq M$ and consider the sequence of measures $\nu_{\varepsilon}$ on $\Omega \times D_{1}$ defined by

$$
\nu_{\varepsilon}:=\left|F_{\varepsilon} \cap \Omega\right|^{-1} u_{\varepsilon}(x) \delta_{y_{\varepsilon}(x)}(d y) d x .
$$

Since the sequence $\nu_{\varepsilon}\left(\Omega \times D_{1}\right)$ is bounded, there exists a measure $\nu$ such that $\nu_{\varepsilon} \rightarrow \nu$ for some subsequence. Moreover, for every $\varphi \in \mathscr{D}$, we have

$$
\begin{aligned}
& \int_{\Omega \times D_{1}} \varphi(x, y) d \nu_{\varepsilon}=\left|F_{\varepsilon} \cap \Omega\right|^{-1} \int_{F_{\varepsilon} \cap \Omega} \varphi\left(x, y_{\varepsilon}(x)\right) u_{\varepsilon}(x) d x \\
& \leq\left(f_{F_{\varepsilon} \cap \Omega}\left(u_{\varepsilon}(x)\right)^{2} d x\right)^{1 / 2}\left(f_{F_{\varepsilon} \cap \Omega}\left(\varphi\left(x, y_{\varepsilon}(x)\right)\right)^{2} d x\right)^{1 / 2} \\
& \leq M^{1 / 2}\left(f_{F_{\varepsilon} \cap \Omega} \varphi\left(x, y_{\varepsilon}(x)\right)^{2} d x\right)^{1 / 2} .
\end{aligned}
$$

As $\varphi^{2} \in \mathscr{D}$, using Lemma 1, we have

$$
\begin{aligned}
\limsup _{\varepsilon \rightarrow 0} & \int_{\Omega \times D_{1}} \varphi(x, y) d \nu_{\varepsilon} \leq M^{1 / 2}\left(f_{\Omega} f_{D_{1}}(\varphi(x, y))^{2} d y d x\right)^{1 / 2} \\
& \int_{\Omega \times D_{1}} \varphi(x, y) d \nu \leq M^{1 / 2} \pi^{-1 / 2}\|\varphi\|_{L^{2}\left(\Omega \times D_{1}\right)} .
\end{aligned}
$$

The measure $\nu$, as a linear functional, is bounded on the unit ball of $\mathrm{L}^{2}\left(\Omega \times D_{1}, \mathbb{R}\right)$ : there exists a function $v \in \mathrm{L}^{2}\left(\Omega \times D_{1}, \mathbb{R}\right)$ such that $\nu=v d x d y$. The convergence of the sequence of measures $\nu_{\varepsilon}$ to the measure $v d x d y$ is clearly equivalent to the d.s.-convergence of $u_{\varepsilon}$ to $v$.

Let us notice that Lemma 2 can obviously be extended to vector or matrix fields.

Lemma 3. Let $u_{\varepsilon}$ be a bounded sequence in $\mathrm{H}^{1}\left(\Omega, \mathbb{R}^{3}\right)$. Then, there exists a constant $C$ such that, for $\varepsilon$ small enough,

$$
f_{F_{\varepsilon} \cap \Omega}\left(u_{\varepsilon}(x)\right)^{2} d x \leq C\left(\left\|u_{\varepsilon}\right\|_{L^{2}\left(\Omega, \mathbb{R}^{3}\right)}-\varepsilon^{2} \log \left(r_{\varepsilon}\right)\right) .
$$

Proof: Assume that $\left\|u_{\varepsilon}\right\|_{\mathrm{H}^{1}\left(\Omega, \mathbb{R}^{3}\right)}^{2} \leq M$. Then

$$
\sum_{p \in \mathscr{P}_{\varepsilon}} \int_{P_{\varepsilon}^{p}}\left(\nabla u_{\varepsilon}\right)^{2} d x \leq M
$$

In each period $P_{\varepsilon}^{p}$, we use the cylindrical coordinates, defining $u_{\varepsilon}^{p}$ by

$$
u_{\varepsilon}^{p}\left(r, \theta, x_{3}\right):=u_{\varepsilon}\left(x_{1}^{p}+r \cos \theta, x_{2}^{p}+r \sin \theta, x_{3}\right) .
$$

Then, we have, for every $\rho_{1} \leq \rho_{2} \leq \varepsilon / 2$,

$$
\sum_{p \in \mathscr{P}_{\varepsilon}} \int_{0}^{1} \int_{0}^{2 \pi} \int_{\rho_{1}}^{\rho_{2}}\left(\frac{\partial u_{\varepsilon}^{p}}{\partial r}\right)^{2} r d r d \theta d x_{3} \leq M
$$

A simple one-dimensional minimization shows that

$$
\int_{\rho_{1}}^{\rho_{2}}\left(\frac{\partial u_{\varepsilon}^{p}}{\partial r}\right)^{2} r d r \geq \frac{\left[u_{\varepsilon}^{p}\left(\rho_{2}\right)-u_{\varepsilon}^{p}\left(\rho_{1}\right)\right]^{2}}{\log \left(\rho_{2}\right)-\log \left(\rho_{1}\right)}
$$

Hence

$$
\sum_{p \in \mathscr{P} \varepsilon} \int_{0}^{1} \int_{0}^{2 \pi}\left[u_{\varepsilon}^{p}\left(\rho_{2}, \theta, x_{3}\right)-u_{\varepsilon}^{p}\left(\rho_{1}, \theta, x_{3}\right)\right]^{2} d \theta d x_{3} \leq M \log \left(\frac{\rho_{2}}{\rho_{1}}\right) .
$$

Let us denote by $f$ the quantity 


$$
f(\rho)=\sum_{p \in \mathscr{P} \varepsilon} \int_{0}^{1} \int_{0}^{2 \pi}\left(u_{\varepsilon}^{p}\left(\rho, \theta, x_{3}\right)\right)^{2} d \theta d x_{3}
$$

The last inequality implies that, for every $\rho_{1} \leq \rho_{2} \leq \varepsilon / 2$,

$$
f\left(\rho_{1}\right) \leq 2 f\left(\rho_{2}\right)+2 M \log \left(\frac{\rho_{2}}{\rho_{1}}\right) .
$$

As the ratio $r_{\varepsilon} / \varepsilon$ tends to zero, we may assume, without loss of generality, that $r_{\varepsilon} \leq \varepsilon / 4$. Then, for every $\rho_{2} \in[\varepsilon / 4, \varepsilon / 2]$, we can bound the mean value of $u_{\varepsilon}^{2}$ on $F_{\varepsilon} \cap \Omega$ by

$$
\begin{aligned}
f_{F_{\varepsilon} \cap \Omega} u_{\varepsilon}^{2} d x & =\left|F_{\varepsilon} \cap \Omega\right|^{-1} \int_{0}^{r_{\varepsilon}} f(r) r d r \\
& \leq\left|F_{\varepsilon} \cap \Omega\right|^{-1} \int_{0}^{r_{\varepsilon}}\left(2 r f\left(\rho_{2}\right)+2 M r \log \left(\frac{\rho_{2}}{r}\right)\right) d r \\
& \leq \frac{\varepsilon^{2}}{\pi r_{\varepsilon}^{2}}\left(f\left(\rho_{2}\right) r_{\varepsilon}^{2}+M r_{\varepsilon}^{2}\left(\log \left(\frac{\rho_{2}}{r_{\varepsilon}}\right)+\frac{1}{2}\right)\right) \\
& \leq \frac{1}{\pi}\left(4 \varepsilon \rho_{2} f\left(\rho_{2}\right)+M \varepsilon^{2}\left(\log \left(\frac{\varepsilon}{r_{\varepsilon}}\right)+\frac{1}{2}\right)\right)
\end{aligned}
$$

and, taking the mean value of this last term for $\rho_{2} \in[\varepsilon / 4, \varepsilon / 2]$, we get

$$
\begin{aligned}
f_{F_{\varepsilon} \cap \Omega} u_{\varepsilon}^{2} d x & \leq \frac{1}{\pi}\left(16 \int_{\frac{\varepsilon}{4}}^{\frac{\varepsilon}{2}} f(r) r d r+M \varepsilon^{2}\left(\log (\varepsilon)-\log \left(r_{\varepsilon}\right)+\frac{1}{2}\right)\right) \\
& \leq \frac{1}{\pi}\left(16\left\|u_{\varepsilon}\right\|_{\mathrm{L}^{2}\left(\Omega, \mathbb{R}^{3}\right)}^{2}+M \varepsilon^{2}\left(\log (\varepsilon)-\log \left(r_{\varepsilon}\right)+\frac{1}{2}\right)\right) .
\end{aligned}
$$

For $\varepsilon$ sufficiently small, $|\log (\varepsilon)+1 / 2| \leq\left|\log \left(r_{\varepsilon}\right)\right|$. The lemma is proved by taking $C=\sup \{16 / \pi, 2 M / \pi\}$.

Lemma 4. Let $u_{\varepsilon}$ be a bounded sequence in $\mathrm{H}^{1}\left(\Omega, \mathbb{R}^{3}\right)$. Then

i) $\int_{F_{\varepsilon} \cap \Omega} u_{\varepsilon}^{2} d x$ is bounded.

ii) If $u_{\varepsilon} \rightarrow u$ in $\mathrm{L}^{2}\left(\Omega, \mathbb{R}^{3}\right)$, then $f_{F_{\varepsilon} \cap \Omega}\left(u_{\varepsilon}(x)-u(x)\right)^{2} d x \rightarrow 0$.

iii) If $u_{\varepsilon} \rightarrow u$ in $\mathrm{L}^{2}\left(\Omega, \mathbb{R}^{3}\right)$ and $u_{\varepsilon} \longrightarrow v$, then

$$
u(x)=\int_{D_{1}} v(x, y) d y, \quad \text { a.e. in } \Omega .
$$

Proof: Assertion (i) is a trivial consequence of Lemma 3 and assumption (6). Here it becomes clear how assumption (6) connects the displacement in the fibers to the displacement in the matrix. Note that, at this point, the boundedness of $\varepsilon^{2} \log \left(r_{\varepsilon}\right)$ should be sufficient.

Assertion (ii) needs the convergence $\varepsilon^{2} \log \left(r_{\varepsilon}\right) \rightarrow 0$. Then one simply must apply Lemma 3 to the sequence $\left(u_{\varepsilon}-u\right)$.

To prove assertion (iii), let us consider for any $\nu>0$, a field $\Phi_{\nu} \in C_{c}^{\infty}\left(\Omega, \mathbb{R}^{3}\right)$ such that $\| \Phi_{\nu}-$ $u \|_{\mathrm{L}^{2}\left(\Omega, \mathbb{R}^{3}\right)}<\nu$. For any $\varphi \in C_{c}^{\infty}\left(\Omega, \mathbb{R}^{3}\right)$ we have

$$
\begin{aligned}
& \left|\lim _{\varepsilon \rightarrow 0} f_{F_{\varepsilon} \cap \Omega}\left(u_{\varepsilon}(x)-\Phi_{\nu}(x)\right) \varphi(x) d x\right| \\
& \leq \lim _{\varepsilon \rightarrow 0}\left(f_{F_{\varepsilon} \cap \Omega}\left|u_{\varepsilon}(x)-\Phi_{\nu}(x)\right|^{2} d x\right)^{1 / 2} \lim _{\varepsilon \rightarrow 0}\left(f_{F_{\varepsilon} \cap \Omega} \varphi(x)^{2} d x\right)^{1 / 2} .
\end{aligned}
$$


Applying Lemma 3 to the sequence $\left|u_{\varepsilon}-\Phi_{\nu}\right|$ shows that this last term is bounded by the norm $\| u_{\varepsilon}(x)-$ $\Phi_{\nu}(x) \|_{\mathrm{L}^{2}\left(\Omega, \mathbb{R}^{3}\right)}$ and therefore is of order $O(\nu)$. Now, passing to the double scale limit, using the definition of $v$ and Lemma 1 , we get

$$
\left|f_{\Omega}\left(f_{D_{1}} v(x, y) d y-\Phi_{\nu}(x)\right) \varphi(x) d x\right| \leq O(\nu) .
$$

Assertion (iii) is proved by recalling that this inequality is valid for every $\nu$.

\subsection{Limits of a sequence with bounded energy}

Lemma 5. Let $u_{\varepsilon}$ be a sequence of $\mathrm{L}^{2}\left(\Omega, \mathbb{R}^{3}\right)$ with bounded energy. Then, up to a subsequence (still denoted by $\left.u_{\varepsilon}\right)$, there exist $v \in \mathrm{L}^{2}\left(\Omega \times D_{1}, \mathbb{R}^{3}\right), w \in \mathrm{L}^{2}\left(\Omega \times D_{1}, \mathbb{R}\right)$ and $\chi \in \mathrm{L}^{2}\left(\Omega \times D_{1}, \mathscr{l l}\right)$ such that

$$
u_{\varepsilon} \longrightarrow v, \quad \frac{u_{\varepsilon 3}}{r_{\varepsilon}} \longrightarrow w, \quad \frac{e\left(u_{\varepsilon}\right)}{r_{\varepsilon}} \longrightarrow \chi
$$

Proof: Assume $E_{\varepsilon}\left(u_{\varepsilon}\right)<M$, then the sequence $u_{\varepsilon}$ is bounded in $\mathrm{H}^{1}\left(\Omega, \mathbb{R}^{3}\right)$, Lemma 4 states that the sequence $f_{F_{\varepsilon} \cap \Omega} u_{\varepsilon}^{2} d x$ is bounded and Lemma 2 implies the existence of $v \in \mathrm{L}^{2}\left(\Omega \times D_{1}, \mathbb{R}^{3}\right)$ such that $u_{\varepsilon} \rightarrow v$. On the other hand, as $\mu_{\varepsilon} \int_{F_{\varepsilon} \cap \Omega} e\left(u_{\varepsilon}\right)^{2} d x<M$, we have

$$
\frac{\mu_{\varepsilon} r_{\varepsilon}^{4}}{\varepsilon^{2}} \pi f_{F_{\varepsilon} \cap \Omega}\left(\frac{1}{r_{\varepsilon}} \frac{\partial u_{\varepsilon 3}}{\partial x_{3}}\right)^{2} d x<M
$$

As any sequence with bounded energy satisfies $u_{\varepsilon 3}\left(x_{1}, x_{2}, 0\right)=0$ a.e. on $\mathscr{B}$, a simple one-dimensional minimization shows that

$$
\int_{0}^{1}\left(\frac{\partial u_{\varepsilon 3}}{\partial x_{3}}\right)^{2} d x_{3} \geq \frac{\pi^{2}}{4} \int_{0}^{1}\left(u_{\varepsilon 3}\right)^{2} d x_{3}, \quad \text { for a.e. }\left(x_{1}, x_{2}\right)
$$

Hence,

$$
\frac{\mu_{\varepsilon} r_{\varepsilon}^{4}}{\varepsilon^{2}} f_{F_{\varepsilon} \cap \Omega}\left(\frac{u_{\varepsilon 3}}{r_{\varepsilon}}\right)^{2} d x \leq \frac{4}{\pi^{3}} M
$$

As $\mu_{\varepsilon} r_{\varepsilon}^{4} / \varepsilon^{2} \rightarrow \mu_{1}$, the sequence $f_{F_{\varepsilon} \cap \Omega}\left(\frac{u_{\varepsilon} 3}{r_{\varepsilon}}\right)^{2} d x$ is bounded: the sequence $u_{\varepsilon 3} / r_{\varepsilon}$ satisfies the assumptions of Lemma 2; the existence of $w$ is assured.

In the same way, from inequality $\left.\mu_{\varepsilon} \int_{F_{\varepsilon} \cap \Omega} e_{(} u_{\varepsilon}\right)^{2} d x<M$, we deduce

$$
\frac{\mu_{\varepsilon} r_{\varepsilon}^{4}}{\varepsilon^{2}} \pi f_{F_{\varepsilon} \cap \Omega}\left(\frac{e\left(u_{\varepsilon}\right)}{r_{\varepsilon}}\right)^{2} d x<M
$$

The sequence $e\left(u_{\varepsilon}\right) / r_{\varepsilon}$ verifies the assumptions of Lemma 2: the existence of $\chi$ is assured.

Lemma 6. Consider a sequence $u_{\varepsilon}$ with bounded energy and converging to some $u$ in $\mathrm{L}^{2}\left(\Omega, \mathbb{R}^{3}\right)$, then

$$
u \in \mathrm{H}^{1}\left(\Omega, \mathbb{R}^{3}\right), \quad \frac{\partial^{2} u_{1}}{\partial x_{3}^{2}} \in \mathrm{L}^{2}\left(\Omega, \mathbb{R}^{3}\right), \quad \frac{\partial^{2} u_{2}}{\partial x_{3}^{2}} \in \mathrm{L}^{2}\left(\Omega, \mathbb{R}^{3}\right), \quad u_{3}(x)=0 \text { a.e. in } \Omega .
$$

Moreover, there exists a subsequence (still denoted by $u_{\varepsilon}$ ) and $q \in \mathrm{L}^{2}(\Omega, \mathbb{R})$ such that

$$
\left(\begin{array}{c}
e\left(u_{\varepsilon}\right) \\
r_{\varepsilon}
\end{array}\right)_{33} \rightarrow q(x)-\frac{\partial^{2} u_{1}}{\partial x_{3}^{2}}(x) y_{1}-\frac{\partial^{2} u_{2}}{\partial x_{3}^{2}}(x) y_{2}
$$


Proof: First, let us notice that the sequence $u_{\varepsilon}$ is bounded in $\mathrm{H}^{1}\left(\Omega, \mathbb{R}^{3}\right)$. Then the limit $u$ belongs to $\mathrm{H}^{1}\left(\Omega, \mathbb{R}^{3}\right)$. Lemma 5 assures the existence of $v \in \mathrm{L}^{2}\left(\Omega \times D_{1}, \mathbb{R}^{3}\right), w \in \mathrm{L}^{2}\left(\Omega \times D_{1}, \mathbb{R}\right)$ and $\chi \in \mathrm{L}^{2}\left(\Omega \times D_{1}, \mathscr{C}\right)$ such that, up to a subsequence,

$$
u_{\varepsilon} \rightarrow v, \quad \frac{u_{\varepsilon 3}}{r_{\varepsilon}} \rightarrow w \quad \text { and } \quad \frac{e\left(u_{\varepsilon}\right)}{r_{\varepsilon}} \rightarrow \chi .
$$

The convergence $u_{\varepsilon 3} / r_{\varepsilon} \longrightarrow w$ immediately yields $u_{\varepsilon 3} \longrightarrow 0$, i.e. $v_{3}=0$. Using the relation $u(x)=f_{D_{1}} v(x, y) d y$ stated in Lemma 4 , we get the identity $u_{3}=0$ a.e. in $\Omega$.

Consider now a tensor field $\varphi \in C_{c}^{\infty}\left(\Omega \times D_{1}, \mathscr{M}\right)$. We have, using the definition of $\chi$ and the divergence theorem,

$$
\begin{aligned}
& f_{\Omega} f_{D_{1}} \chi_{i j}(x, y) \varphi_{i j}(x, y) d y d x=\lim _{\varepsilon \rightarrow 0} f_{F_{\varepsilon} \cap \Omega} \frac{1}{r_{\varepsilon}} e_{i j}\left(u_{\varepsilon}\right)(x) \varphi_{i j}\left(x, y_{\varepsilon}(x)\right) d x, \\
& =\lim _{\varepsilon \rightarrow 0} f_{F_{\varepsilon} \cap \Omega} \frac{1}{r_{\varepsilon}} \frac{\partial u_{\varepsilon i}}{\partial x_{j}}(x) \varphi_{i j}\left(x, y_{\varepsilon}(x)\right) d x, \\
& =-\lim _{\varepsilon \rightarrow 0} f_{F_{\varepsilon} \cap \Omega} \frac{1}{r_{\varepsilon}} u_{\varepsilon i}(x)\left[\frac{\partial \varphi_{i j}}{\partial x_{j}}\left(x, y_{\varepsilon}(x)\right)+\frac{1}{r_{\varepsilon}} \frac{\partial \varphi_{i \alpha}}{\partial y_{\alpha}}\left(x, y_{\varepsilon}(x)\right)\right] d x \text {, } \\
& =-\lim _{\varepsilon \rightarrow 0}\left\{\frac{1}{r_{\varepsilon}^{2}} f_{F_{\varepsilon} \cap \Omega} u_{\varepsilon \beta}(x) \frac{\partial \varphi_{\beta \alpha}}{\partial y_{\alpha}}\left(x, y_{\varepsilon}(x)\right) d x\right. \\
& +\frac{1}{r_{\varepsilon}} f_{F_{\varepsilon} \cap \Omega}\left(u_{\varepsilon \beta}(x) \frac{\partial \varphi_{\beta j}}{\partial x_{j}}\left(x, y_{\varepsilon}(x)\right)+\frac{u_{\varepsilon 3}(x)}{r_{\varepsilon}} \frac{\partial \varphi_{3 \alpha}}{\partial y_{\alpha}}\left(x, y_{\varepsilon}(x)\right)\right) d x \\
& \left.+f_{F_{\varepsilon} \cap \Omega} \frac{u_{\varepsilon 3}(x)}{r_{\varepsilon}} \frac{\partial \varphi_{3 j}}{\partial x_{j}}\left(x, y_{\varepsilon}(x)\right) d x\right\} .
\end{aligned}
$$

Multiplying equation (23) by $r_{\varepsilon}^{2}$ and passing to the limit $\varepsilon \rightarrow 0$ gives

$$
\begin{gathered}
\lim _{\varepsilon \rightarrow 0} f_{F_{\varepsilon} \cap \Omega} u_{\varepsilon \beta}(x) \frac{\partial \varphi_{\beta \alpha}}{\partial y_{\alpha}}\left(x, y_{\varepsilon}(x)\right) d x=0 \\
f_{\Omega} f_{D_{1}} v_{\beta}(x, y) \frac{\partial \varphi_{\beta \alpha}}{\partial y_{\alpha}}(x, y) d y d x=0 \\
\left\langle\frac{\partial v_{\beta}}{\partial y_{\alpha}} \varphi_{\beta \alpha}\right\rangle=0
\end{gathered}
$$

where $<>$ denotes the distribution bracket on $\Omega \times D_{1}$. This last equation, valid for any field $\varphi$ of a symmetric plane matrix and whose support is included in $\Omega \times D_{1}$, is equivalent to the antisymmetry (in the sense of distributions)

$$
\frac{\partial v_{1}}{\partial y_{2}}=-\frac{\partial v_{2}}{\partial y_{1}}, \quad \frac{\partial v_{1}}{\partial y_{1}}=\frac{\partial v_{2}}{\partial y_{2}}=0 .
$$

Then (refer for instance to [15]) there exist three functions $c_{1}, c_{2}$ and $t$ in $\mathrm{L}^{2}(\Omega, \mathbb{R})$ such that

$$
v_{1}(x, y)=c_{1}(x)-t(x) y_{2}, \quad v_{2}(x, y)=c_{2}(x)+t(x) y_{1} .
$$

Lemma 4 implies $c_{1}=u_{1}$ and $c_{2}=u_{2}$. Hence

$$
v_{1}(x, y)=u_{1}(x)-t(x) y_{2}, \quad v_{2}(x, y)=u_{2}(x)+t(x) y_{1} .
$$

Now, consider the fields $\varphi$ such that $\varphi_{\beta \alpha}=0, \forall \alpha, \beta \in\{1,2\}$. Multiplying equation (23) by $r_{\varepsilon}$ and passing to the limit gives 


$$
\begin{gathered}
\lim _{\varepsilon \rightarrow 0} f_{F_{\varepsilon} \cap \Omega}\left[u_{\varepsilon \beta}(x) \frac{\partial \varphi_{\beta 3}}{\partial x_{3}}\left(x, y_{\varepsilon}(x)\right)+\frac{\left.u_{\varepsilon}(x) \frac{\partial \varphi_{3 \alpha}}{\partial y_{\alpha}}\left(x, y_{\varepsilon}(x)\right)\right] d x=0}{r_{\varepsilon}}\right. \\
f_{\Omega} f_{D_{1}}\left[v_{\beta}(x, y) \frac{\partial \varphi_{\beta 3}}{\partial x_{3}}(x, y)+w(x, y) \frac{\partial \varphi_{3 \alpha}}{\partial y_{\alpha}}(x, y)\right] d y d x=0 \\
\left\langle\frac{\partial v_{\beta}}{\partial x_{3}} \varphi_{\beta 3}\right\rangle+\left\langle\frac{\partial w}{\partial y_{\alpha}} \varphi_{3 \alpha}\right\rangle=0 \\
\left\langle\left(\frac{\partial v_{\alpha}}{\partial x_{3}}+\frac{\partial w}{\partial y_{\alpha}}\right) \varphi_{3 \alpha}\right\rangle=0
\end{gathered}
$$

This last equation, valid for every functions $\varphi_{3 \alpha}$ whose support is included in $\Omega \times D_{1}$, implies that, in the sense of distributions,

$$
\frac{\partial v_{\alpha}}{\partial x_{3}}+\frac{\partial w}{\partial y_{\alpha}}=0
$$

which, using (25), becomes

$$
-\frac{\partial u_{1}}{\partial x_{3}}+\frac{\partial t}{\partial x_{3}} y_{2}=\frac{\partial w}{\partial y_{1}}, \quad-\frac{\partial u_{2}}{\partial x_{3}}-\frac{\partial t}{\partial x_{3}} y_{1}=\frac{\partial w}{\partial y_{2}} .
$$

The Schwarz theorem implies that $\partial t / \partial x_{3}=0$; then

$$
-\frac{\partial u_{1}}{\partial x_{3}}=\frac{\partial w}{\partial y_{1}}, \quad-\frac{\partial u_{2}}{\partial x_{3}}=\frac{\partial w}{\partial y_{2}}
$$

Therefore there exists a function $s$ in $\mathrm{L}^{2}(\Omega, \mathbb{R})$ such that

$$
w(x, y)=-\frac{\partial u_{\alpha}}{\partial x_{3}} y_{\alpha}+s(x)
$$

Finally, considering matrix fields $\varphi$ with a unique non vanishing component $\varphi_{33}$, equation (23) leads to

$$
\begin{aligned}
f_{\Omega} f_{D_{1}} \chi_{33}(x, y) \varphi_{33}(x, y) d x & =-\lim _{\varepsilon \rightarrow 0} f_{F_{\varepsilon} \cap \Omega} \frac{u_{\varepsilon 3}}{r_{\varepsilon}}(x) \frac{\partial \varphi_{33}}{\partial x_{3}}\left(x, y_{\varepsilon}(x)\right) d x \\
& =-f_{\Omega} f_{D_{1}} w(x, y) \frac{\partial \varphi_{33}}{\partial x_{3}}(x, y) d y d x \\
\left\langle\chi_{33} \varphi_{33}\right\rangle & =\left\langle\frac{\partial w}{\partial x_{3}} \varphi_{33}\right\rangle .
\end{aligned}
$$

Then $\chi_{33}=\partial w / \partial x_{3}$ in the sense of distributions. As $\chi$ belongs to $\mathrm{L}^{2}\left(\Omega \times D_{1}, \mathscr{\ell}\right), \partial w / \partial x_{3}$ belongs to $\mathrm{L}^{2}\left(\Omega \times D_{1}, \mathbb{R}\right)$. This means, by using (27) that $\partial^{2} u_{\alpha} / \partial x_{3}^{2} \in \mathrm{L}^{2}(\Omega, \mathbb{R}), q:=\partial s / \partial x_{3} \in \mathrm{L}^{2}(\Omega, \mathbb{R})$ and

$$
\chi_{33}(x, y)=-\frac{\partial^{2} u_{\alpha}}{\partial x_{3}^{2}}(x) y_{\alpha}+q(x) .
$$

\subsection{Lower bound for the energy}

Let $u_{\varepsilon}$ be a sequence with bounded energy converging to some $u$ in $\mathrm{L}^{2}\left(\Omega, \mathbb{R}^{3}\right)$. We can assume without loss of generality that $E_{\varepsilon}\left(u_{\varepsilon}\right)$ converges to $\lim \inf E_{\varepsilon}\left(u_{\varepsilon}\right)$. Then assertion (ii) of Theorem 1 will be proved if we prove that for some subsequence (still denoted by $u_{\varepsilon}$ ) we have

$$
\liminf _{\varepsilon \rightarrow 0} E_{\varepsilon}\left(u_{\varepsilon}\right) \geq E_{0}(u)
$$

First, let us recall that the sequence $u_{\varepsilon}$ is bounded in $\mathrm{H}^{1}\left(\Omega, \mathbb{R}^{3}\right)$, then 


$$
u \in \mathrm{H}^{1}\left(\Omega, \mathbb{R}^{3}\right)
$$

It is easy to get the lower bound for the energy outside the fibers: indeed, as $E_{\varepsilon}\left(u_{\varepsilon}\right)$ is bounded, $E_{\varepsilon}^{f}\left(F_{\varepsilon} \cap \Omega, u_{\varepsilon}\right)$ is also bounded. As the ratios $\mu_{0} / \mu_{\varepsilon}$ and $\lambda_{0} / \lambda_{\varepsilon}$ tend to zero, then $E^{m}\left(F_{\varepsilon} \cap \Omega, u_{\varepsilon}\right)$ tends to 0 . Hence

$$
\liminf _{\varepsilon \rightarrow 0} E^{m}\left(M_{\varepsilon} \cap \Omega, u_{\varepsilon}\right)=\liminf _{\varepsilon \rightarrow 0} E^{m}\left(\Omega, u_{\varepsilon}\right) \geq E^{m}(\Omega, u) .
$$

To estimate the energy in the fibers we use the lemmas stated in the preceeding subsections. Indeed we have

$$
\begin{aligned}
\liminf _{\varepsilon \rightarrow 0} & E_{\varepsilon}^{f}\left(F_{\varepsilon} \cap \Omega, u_{\varepsilon}\right) \\
& =\liminf _{\varepsilon \rightarrow 0}\left\{\frac{\mu_{\varepsilon} r_{\varepsilon}^{4}}{\varepsilon^{2}} \pi f_{F_{\varepsilon} \cap \Omega}\left(\frac{e\left(u_{\varepsilon}\right)}{r_{\varepsilon}}\right)^{2}+\frac{\lambda_{\varepsilon}}{\mu_{\varepsilon}}\left(\frac{\operatorname{Tr}\left(e\left(u_{\varepsilon}\right)\right)}{r_{\varepsilon}}\right)^{2} d x\right\} \\
& \geq \pi \mu_{1} \liminf _{\varepsilon \rightarrow 0} f_{F_{\varepsilon} \cap \Omega}\left[\left(\frac{e\left(u_{\varepsilon}\right)}{r_{\varepsilon}}\right)^{2}+\frac{\ell}{2}\left(\frac{\operatorname{Tr}\left(e\left(u_{\varepsilon}\right)\right)}{r_{\varepsilon}}\right)^{2}\right] d x
\end{aligned}
$$

From Lemma 5, we know that, possibly passing to a subsequence, $e\left(u_{\varepsilon}\right) / r_{\varepsilon}$ admits a double scale limit $\chi$. As we cannot pass to the limit directly in inequality (32), we write its dual form

$$
\begin{aligned}
\liminf _{\varepsilon \rightarrow 0} E_{\varepsilon}^{f}\left(F_{\varepsilon} \cap \Omega, u_{\varepsilon}\right) \geq \sup _{\varphi}\left\{\pi \mu _ { 1 } \operatorname { l i m i n f } _ { \varepsilon \rightarrow 0 } f _ { F _ { \varepsilon } \cap \Omega } \left[\frac{e\left(u_{\varepsilon}(x)\right)}{r_{\varepsilon}}: \varphi\left(x, y_{\varepsilon}(x)\right)-\right.\right. \\
\\
\left.\left.\frac{1}{4} \varphi\left(x, y_{\varepsilon}(x)\right)^{2}+\frac{\ell}{4(2+3 \ell)}\left(\operatorname{Tr}\left(\varphi\left(x, y_{\varepsilon}(x)\right)\right)\right)^{2}\right] d x\right\},
\end{aligned}
$$

where the supremum is taken for every $\varphi \in C_{c}^{\infty}\left(\Omega \times D_{1}, \mathscr{C l}\right)$. Then Remark 1 and Lemma 1 allow to pass to the limit

$$
\begin{aligned}
\liminf _{\varepsilon \rightarrow 0} & E_{\varepsilon}^{f}\left(F_{\varepsilon} \cap \Omega, u_{\varepsilon}\right) \geq \sup _{\varphi}\left\{\pi \mu_{1} f_{\Omega} f_{D_{1}}[\chi(x, y): \varphi(x, y)-\right. \\
& \left.\left.\frac{1}{4}(\varphi(x, y))^{2}+\frac{\ell}{4(2+3 \ell)}(\operatorname{Tr}(\varphi(x, y)))^{2}\right] d y d x\right\} .
\end{aligned}
$$

As $C_{c}^{\infty}\left(\Omega \times D_{1}, \mathscr{C}\right)$ is dense in $\mathrm{L}^{2}\left(\Omega \times D_{1}, \mathscr{C l}\right)$, we get

$$
\liminf _{\varepsilon \rightarrow 0} E_{\varepsilon}^{f}\left(F_{\varepsilon} \cap \Omega, u_{\varepsilon}\right) \geq \pi \mu_{1} f_{\Omega} f_{D_{1}}\left[\chi^{2}(x, y)+\frac{\ell}{2}(\operatorname{Tr}(\chi(x, y)))^{2}\right] d y d x .
$$

It is easy to verify that, for every $M$ in $\mathscr{M}$,

$$
M^{2}+\frac{\ell}{2}(\operatorname{Tr}(M))^{2} \geq \frac{3 \ell+2}{2(\ell+1)} M_{33}^{2} .
$$

Hence,

$$
\liminf _{\varepsilon \rightarrow 0} E_{\varepsilon}^{f}\left(F_{\varepsilon} \cap \Omega, u_{\varepsilon}\right) \geq \pi \mu_{1} \frac{3 \ell+2}{(\ell+1)} f_{\Omega} f_{D_{1}} \chi_{33}^{2}(x, y) d y d x
$$

From Lemma 6, we know that

$$
\frac{\partial^{2} u_{\alpha}}{\partial x_{3}^{2}} \in \mathrm{L}^{2}(\Omega, \mathbb{R})
$$

and we can express $\chi_{33}$ in terms of these second derivatives of $u$

$$
\liminf _{\varepsilon \rightarrow 0} E_{\varepsilon}^{f}\left(F_{\varepsilon} \cap \Omega, u_{\varepsilon}\right) \geq \frac{\pi}{2} \mu_{1} \frac{3 \ell+2}{(\ell+1)} f_{\Omega} f_{D_{1}}\left(q(x)-\frac{\partial^{2} u_{\alpha}}{\partial x_{3}^{2}}(x) y_{\alpha}\right)^{2} d y d x
$$

For $\alpha=1$ or 2 , we have

$$
f_{D_{1}} y_{\alpha} d y=0, \quad f_{D_{1}} y_{\alpha}^{2} d y=\frac{1}{4}, \quad \text { and } \quad f_{D_{1}} y_{1} y_{2} d y=0 .
$$


Then we may deduce that

$$
\liminf _{\varepsilon \rightarrow 0} E_{\varepsilon}^{f}\left(F_{\varepsilon} \cap \Omega, u_{\varepsilon}\right) \geq \frac{\pi \mu_{1}(3 \ell+2)}{8(\ell+1)} f_{\Omega}\left[4 q^{2}(x)+\left(\frac{\partial^{2} u_{1}}{\partial x_{3}^{2}}\right)^{2}+\left(\frac{\partial^{2} u_{2}}{\partial x_{3}^{2}}\right)^{2}\right] d x
$$

which implies

$$
\liminf _{\varepsilon \rightarrow 0} E_{\varepsilon}^{f}\left(F_{\varepsilon} \cap \Omega, u_{\varepsilon}\right) \geq \frac{k}{2} f_{\Omega}\left[\left(\frac{\partial^{2} u_{1}}{\partial x_{3}^{2}}\right)^{2}+\left(\frac{\partial^{2} u_{2}}{\partial x_{3}^{2}}\right)^{2}\right] d x
$$

where $k$ is defined by (10).

In order to obtain the boundary conditions, let us consider the extended domain $\left.\tilde{\Omega}:=(0,1)^{2} \times\right]-1,1[$ and the extensions $\tilde{u}_{\varepsilon}$ and $\tilde{u}$ of $u_{\varepsilon}$ and $u$ on $\tilde{\Omega}$ defined by

$$
\begin{gathered}
\tilde{u}_{\varepsilon}:=u_{\varepsilon} \text { on } \Omega, \quad \tilde{u}:=u \text { on } \Omega, \\
\tilde{u}_{\varepsilon}:=\tilde{u}:=0 \text { on } \tilde{\Omega} \backslash \Omega .
\end{gathered}
$$

The sequence $E_{\varepsilon}\left(\tilde{\Omega}, \tilde{u}_{\varepsilon}\right)$ is bounded and $\tilde{u}_{\varepsilon}$ converges to $\tilde{u}$ in $\mathrm{L}^{2}\left(\tilde{\Omega}, \mathbb{R}^{3}\right)$; thus the results of Lemma 6 can be applied: $\tilde{u} \in \mathrm{H}^{1}\left(\tilde{\Omega}, \mathbb{R}^{3}\right)$ and $\partial^{2} \tilde{u}_{\alpha} / \partial x_{3}^{2} \in \mathrm{L}^{2}(\tilde{\Omega}, \mathbb{R})$ which implies

$$
u=0 \text { a.e. on } \mathscr{B}, \quad \frac{\partial u_{1}}{\partial x_{3}}=\frac{\partial u_{2}}{\partial x_{3}}=0 \text { a.e. on } \mathscr{B} .
$$

Assertion (ii) of Theorem 1 is proved by recalling (30), (31), (35), (36), and (37).

\section{Proof of the upper bound inequality}

Let us denote by $\mathscr{H}$ the functional space

$$
\left\{u \in \mathrm{H}^{1}\left(\Omega, \mathbb{R}^{3}\right), u_{3}=0 \text { a.e. on } \Omega, \frac{\partial^{2} u}{\partial x_{3}^{2}} \in \mathrm{L}^{2}\left(\Omega, \mathbb{R}^{3}\right), u=\frac{\partial u}{\partial x_{3}}=0 \text { a.e. on } \mathscr{B}\right\},
$$

which is endowed with the norm

$$
\|u\|_{\mathscr{H}}:=\|u\|_{\mathrm{H}^{1}\left(\Omega, \mathbb{R}^{3}\right)}+\left\|\frac{\partial^{2} u}{\partial x_{3}^{2}}\right\|_{\mathrm{L}^{2}\left(\Omega, \mathbb{R}^{3}\right)} .
$$

For any $u \in \mathrm{L}^{2}\left(\Omega, \mathbb{R}^{3}\right)$ such that $E_{0}(u)<+\infty$, i.e., for any $u \in \mathscr{H}$, we have to construct an approximating sequence $u_{\varepsilon}$ in $\mathrm{L}^{2}\left(\Omega, \mathbb{R}^{3}\right)$ such that

$$
u_{\varepsilon} \longrightarrow u \text { in } \mathrm{L}^{2}\left(\Omega, \mathbb{R}^{3}\right) \text { and } \limsup _{\varepsilon \longrightarrow 0} E_{\varepsilon}\left(u_{\varepsilon}\right) \leq E_{0}(u)
$$

It is easy to verify that

$$
\mathscr{\mathscr { B }}:=\left\{u \in C^{\infty}\left(\Omega, \mathbb{R}^{3}\right), u=\frac{\partial u}{\partial x_{3}}=\frac{\partial^{2} u}{\partial x_{3}^{2}}=0 \text { a.e. on } \mathscr{B}\right\}
$$

is dense in $\mathscr{H} \mathscr{C}$. Then, we can restrict our study to a function $u \in \mathscr{\mathscr { H }}$. As $E_{0}$ is continuous on $\mathscr{H}$, the result can be generalized to $\mathscr{H}$.

Let us choose a sequence $R_{\varepsilon}$ such that $r_{\varepsilon}<<R_{\varepsilon}<<\varepsilon$, and let us divide $M_{\varepsilon}$ in two parts by introducing a transition layer $C_{\varepsilon}$

$$
C_{\varepsilon}:=\left\{x \in \Omega: 1<\left|y_{\varepsilon}(x)\right|<r_{\varepsilon}^{-1} R_{\varepsilon}\right\}, \quad B_{\varepsilon}:=\left\{x \in \Omega:\left|y_{\varepsilon}(x)\right|>r_{\varepsilon}^{-1} R_{\varepsilon}\right\},
$$

The part of $C_{\varepsilon}$ contained in a period $P_{\varepsilon}^{p}$ is denoted by $C_{\varepsilon}^{p}:=C_{\varepsilon} \cap P_{\varepsilon}^{p}$

For every $p$ in $\mathscr{P}_{\varepsilon}$, we define the function $v_{\varepsilon}^{p} \in C^{\infty}\left((0,1), \mathbb{R}^{3}\right)$ by 


$$
v_{\varepsilon}^{p}\left(x_{3}\right):=\int_{D_{1}} u\left(x_{1}^{p}+r_{\varepsilon} y_{1}, x_{2}^{p}+r_{\varepsilon} y_{2}, x_{3}\right) d y_{1} d y_{2}
$$

and the function $w_{\varepsilon}^{p} \in C^{\infty}\left((0,1) \times \mathbb{R}^{2}, \mathbb{R}^{3}\right)$ by

$$
\begin{aligned}
& w_{\varepsilon 1}^{p}\left(x_{3}, y\right):=v_{\varepsilon 1}^{p}\left(x_{3}\right)+r_{\varepsilon}^{2} \frac{\ell}{2(\ell+1)}\left[\begin{array}{cc}
\partial^{2} v_{\varepsilon 1}^{p} & y_{1}^{2}-y_{2}^{2} \\
\partial x_{3}^{2} & 2
\end{array}+\frac{\partial^{2} v_{\varepsilon 2}^{p}}{\partial x_{3}^{2}} y_{1} y_{2}\right] \text {, } \\
& w_{\varepsilon 2}^{p}\left(x_{3}, y\right):=v_{\varepsilon 2}^{p}\left(x_{3}\right)+r_{\varepsilon}^{2} \frac{\ell}{2(\ell+1)}\left[\begin{array}{cc}
\partial^{2} v_{\varepsilon 2}^{p} y_{2}^{2}-y_{1}^{2} \\
\partial x_{3}^{2} & 2
\end{array} \frac{\partial^{2} v_{\varepsilon 1}^{p}}{\partial x_{3}^{2}} y_{1} y_{2}\right] \text {, } \\
& w_{\varepsilon 3}^{p}\left(x_{3}, y\right):=-r_{\varepsilon} \frac{\partial v_{\varepsilon \alpha}^{p}}{\partial x_{3}} y_{\alpha} .
\end{aligned}
$$

The function $w_{\varepsilon}^{p}$ may be interpreted as the rod-like displacement of the fiber $F_{\varepsilon}^{p}$ whose global displacement is $v_{\varepsilon}^{p}$ [3]. As $u \in \mathscr{\mathscr { H }}$, we have $u=\partial u / \partial x_{3}=\partial^{2} u / \partial x_{3}^{2}=0$ on $\mathscr{B}$. Therefore every fonction $w_{\varepsilon}^{p}$ vanishes for $x_{3}=0$.

We define now the approximating sequence $\left(u_{\varepsilon}\right)$ by setting

$$
u_{\varepsilon}(x):=\left\{\begin{aligned}
u(x) & \text { on } B_{\varepsilon}, \\
w_{\varepsilon}^{p}\left(x_{3}, y_{\varepsilon}(x)\right) & \text { on each fiber } F_{\varepsilon}^{p}, \\
\gamma(r) w_{\varepsilon}^{p}\left(x_{3},(\cos \theta, \sin \theta)\right) & \text { on each transition } \\
+(1-\gamma(r)) u(x) & \text { layer } C_{\varepsilon}^{p},
\end{aligned}\right.
$$

where $(r, \theta)$ denote the polar coordinates defined in each period $P_{\varepsilon}^{p}$ by $x_{1}=x_{1}^{p}+r \cos \theta, x_{2}=x_{2}^{p}+r \sin \theta$ and $\gamma$ is the function defined by

$$
\gamma(r):=\frac{\log (r)-\log \left(R_{\varepsilon}\right)}{\log \left(r_{\varepsilon}\right)-\log \left(R_{\varepsilon}\right)} .
$$

Notice that, by construction, $u_{\varepsilon}$ belongs to $\mathrm{H}^{1}\left(\Omega, \mathbb{R}^{3}\right)$ and satisfies $u_{\varepsilon}=0$ on $\mathscr{B}$. Then

$$
E_{\varepsilon}\left(u_{\varepsilon}\right)=E^{m}\left(B_{\varepsilon} \cap \Omega, u_{\varepsilon}\right)+E^{m}\left(C_{\varepsilon} \cap \Omega, u_{\varepsilon}\right)+E_{\varepsilon}^{f}\left(F_{\varepsilon} \cap \Omega, u_{\varepsilon}\right) .
$$

Moreover, $u_{\varepsilon}$ tends to $u$ in $\mathrm{L}^{2}\left(\Omega, \mathbb{R}^{3}\right)$ : indeed $u_{\varepsilon}$ coincides with $u$ on $B_{\varepsilon},\left|\Omega \backslash B_{\varepsilon}\right| \rightarrow 0$, and $\left(u_{\varepsilon}\right)$ is uniformly bounded on $F_{\varepsilon}$ and $C_{\varepsilon}$.

\subsection{Estimation for the energy of $u_{\varepsilon}$ in the matrix}

As $u_{\varepsilon}(x):=u(x)$ on $B_{\varepsilon}$, we have $E^{m}\left(B_{\varepsilon}, u_{\varepsilon}\right)=E^{m}\left(B_{\varepsilon}, u\right)$. As $R_{\varepsilon} / \varepsilon \rightarrow 0$ one has $\left|\Omega \backslash B_{\varepsilon}\right| \rightarrow 0$. Moreover, $u \in \mathrm{H}^{1}(\Omega)$, then $E^{m}\left(\Omega \backslash B_{\varepsilon}, u\right) \rightarrow 0$ and

$$
\lim _{\varepsilon \rightarrow 0} E^{m}\left(B_{\varepsilon}, u_{\varepsilon}\right)=E^{m}(\Omega, u)
$$

\subsection{Estimation for the energy of $u_{\varepsilon}$ in the fibers}

Let us estimate the energy of $u_{\varepsilon}$ in each fiber $F_{\varepsilon}^{p}$ : As $u_{\varepsilon}(x)=w_{\varepsilon}^{p}\left(x, y_{\varepsilon}(x)\right)$ in $F_{\varepsilon}^{p}$, we have

$$
\begin{aligned}
& e_{11}\left(u_{\varepsilon}\right)=e_{22}\left(u_{\varepsilon}\right)=r_{\varepsilon} \frac{\ell}{2(\ell+1)} \frac{\partial^{2} v_{\varepsilon \alpha}^{p}}{\partial x_{3}^{2}} y_{\alpha}, \\
& e_{33}\left(u_{\varepsilon}\right)=-r_{\varepsilon} \frac{\partial^{2} v_{\varepsilon \alpha}^{p}}{\partial x_{3}^{2}} y_{\alpha} \\
& e_{12}\left(u_{\varepsilon}\right)=e_{21}\left(u_{\varepsilon}\right)=0 \text {, } \\
& e_{13}\left(u_{\varepsilon}\right)=e_{31}\left(u_{\varepsilon}\right)=r_{\varepsilon}^{2} \frac{\ell}{4(\ell+1)}\left[\begin{array}{cc}
\partial^{3} v_{\varepsilon 1}^{p} & y_{1}^{2}-y_{2}^{2} \\
\partial x_{3}^{3} & 2
\end{array}\right. \\
& e_{23}\left(u_{\varepsilon}\right)=e_{32}\left(u_{\varepsilon}\right)=r_{\varepsilon}^{2} \frac{\ell}{4(\ell+1)}\left[\begin{array}{cc}
\partial^{3} v_{\varepsilon 2}^{p} & y_{2}^{2}-y_{1}^{2} \\
\partial x_{3}^{3} & 2
\end{array}\right.
\end{aligned}
$$


Hence

$$
\begin{aligned}
& E_{\varepsilon}^{f}\left(F_{\varepsilon}^{p}, u_{\varepsilon}\right)=r_{\varepsilon}^{4} \int_{0}^{1} \int_{D_{1}}\left[\mu_{\varepsilon} \frac{3 \ell^{2}+4 \ell+2}{2(\ell+1)^{2}}+\lambda_{\varepsilon} \frac{1}{2(\ell+1)^{2}}\right]\left[\frac{\partial^{2} v_{\varepsilon \alpha}^{p}}{\partial x_{3}^{2}} y_{\alpha}\right]^{2} d x \\
& +r_{\varepsilon}^{6} \int_{0}^{1} \int_{D_{1}}\left[\mu_{\varepsilon} \frac{\ell^{2}}{8(\ell+1)^{2}}\right]\left[\left(\frac{\partial^{3} v_{\varepsilon 1}^{p}}{\partial x_{3}^{3}}\right)^{2}+\left(\frac{\partial^{3} v_{\varepsilon 2}^{p}}{\partial x_{3}^{3}}\right)^{2}\right]\left[\frac{y_{1}^{2}+y_{2}^{2}}{2}\right]^{2} d x .
\end{aligned}
$$

Computing the integrals on $D_{1}$ and summing for all sets $F_{\varepsilon}^{p}$ we get

$$
\begin{gathered}
E_{\varepsilon}^{f}\left(F_{\varepsilon} \cap \Omega, u_{\varepsilon}\right)=\sum_{p \in \mathscr{P} \varepsilon}\left\{\begin{array}{c}
\ell^{2} \pi r_{\varepsilon}^{6} \mu_{\varepsilon} \\
96(\ell+1)^{2}
\end{array} \int_{0}^{1}\left(\left(\frac{\partial^{3} v_{\varepsilon 1}^{p}}{\partial x_{3}^{3}}\right)^{2}+\left(\frac{\partial^{3} v_{\varepsilon 2}^{p}}{\partial x_{3}^{3}}\right)^{2}\right) d x_{3}\right. \\
+\frac{\left.3 \ell^{2}+4 \ell+2+\frac{\lambda_{\varepsilon}}{\mu_{\varepsilon}} \frac{\pi r_{\varepsilon}^{4} \mu_{\varepsilon}}{4} \int_{0}^{1}\left(\left(\frac{\partial^{2} v_{\varepsilon 1}^{p}}{\partial x_{3}^{2}}\right)^{2}+\left(\frac{\partial^{2} v_{\varepsilon 2}^{p}}{\partial x_{3}^{2}}\right)^{2}\right) d x_{3}\right\} .}{2(\ell+1)^{2}} .
\end{gathered}
$$

Passing to the limit $\varepsilon \rightarrow 0$, we have

$$
\varepsilon^{-2} \frac{\ell^{2}}{8(\ell+1)^{2}} \quad \frac{\pi r_{\varepsilon}^{6} \mu_{\varepsilon}}{12} \rightarrow 0, \quad \varepsilon^{-2} \frac{3 \ell^{2}+4 \ell+2+\frac{\lambda_{\varepsilon}}{\mu_{\varepsilon}} \pi r_{\varepsilon}^{4} \mu_{\varepsilon}}{2(\ell+1)^{2}} \rightarrow \frac{k}{2},
$$

where $k$ is defined by (10). Moreover, using the definition of the functions $v_{\varepsilon}^{p}$, we have

$$
\begin{aligned}
\varepsilon^{2} \sum_{p \in \mathscr{P} \varepsilon} \int_{0}^{1}\left(\frac{\partial^{2} v_{\varepsilon \alpha}^{p}}{\partial x_{3}^{2}}\right)^{2} d x_{3} & =\varepsilon^{2} f_{F_{\varepsilon} \cap \Omega}\left(\frac{\partial^{2} u_{\alpha}}{\partial x_{3}^{2}}\right)^{2} d x \\
\varepsilon^{2} \sum_{p \in \mathscr{P}_{\varepsilon}} \int_{0}^{1}\left(\frac{\partial^{3} v_{\varepsilon \alpha}^{p}}{\partial x_{3}^{3}}\right)^{2} d x_{3} & =\varepsilon^{2} f_{F_{\varepsilon} \cap \Omega}\left(\frac{\partial^{3} u_{\alpha}}{\partial x_{3}^{3}}\right)^{2} d x .
\end{aligned}
$$

Hence

$$
\lim _{\varepsilon \rightarrow 0} E_{\varepsilon}^{f}\left(F_{\varepsilon} \cap \Omega, u_{\varepsilon}\right)=\frac{k}{2} f_{\Omega}\left(\frac{\partial^{2} u_{1}^{2}}{\partial x_{3}^{2}}+\frac{\partial^{2} u_{2}^{2}}{\partial x_{3}^{2}}\right) d x
$$

\subsection{Estimation for the energy of $u_{\varepsilon}$ in the transition layer}

Let $M=\sup _{\Omega}\left\{\sup \left(\nabla u, \nabla^{2} u, \nabla^{3} u\right)\right\}$. We restrict attention to a cylinder $C_{\varepsilon}^{p}$ and prove, in a first step, that $\left|\nabla u_{\varepsilon}\right|$ is bounded on $C_{\varepsilon}^{p}$. We use the cylindrical coordinates $\left(r, \theta, x_{3}\right)$ defined by $x_{1}=x_{1}^{p}+r \cos \theta$, $x_{2}=x_{2}^{p}+r \sin \theta$ (on $C_{\varepsilon}^{p}$ we have $r \in\left[r_{\varepsilon}, R_{\varepsilon}\right]$ ).

Clearly, in view of the definition of $w_{\varepsilon}^{p}$, there exists a positive real $M_{1}$ such that

$$
\left|\frac{\partial w_{\varepsilon}^{p}}{\partial \theta}\left(x_{3},(\cos \theta, \sin \theta)\right)\right| \leq M_{1} r_{\varepsilon} .
$$

Moreover, $\left|\frac{\partial u}{\partial \theta}\right| \leq M r$ and whence

$$
\begin{aligned}
\left|\frac{1}{r} \frac{\partial u_{\varepsilon}}{\partial \theta}\right| & =\frac{1}{r} \mid(1-\gamma(r)) \frac{\partial u}{\partial \theta}\left(x_{1}^{p}+r \cos \theta, x_{2}^{p}+r \sin \theta, x_{3}\right)+ \\
& \gamma(r) \frac{\partial w_{\varepsilon}^{p}}{\partial \theta}\left(x_{3},(\cos \theta, \sin \theta)\right) \mid \leq M_{1}+M
\end{aligned}
$$

On the other hand, owing to the definition of $w_{\varepsilon}^{p}$, there exists a positive real $M_{2}$ such that, for every $y \in D_{1}$ and $x_{3} \in[0,1],\left|w_{\varepsilon}^{p}\left(x_{3}, y\right)-v_{\varepsilon}^{p}\left(x_{3}\right)\right| \leq M_{2} r_{\varepsilon}$. From the definition of $v_{\varepsilon}^{p}$, we have, for every $r \geq r_{\varepsilon}$

$$
\left|v_{\varepsilon}^{p}\left(x_{3}\right)-u\left(x_{1}^{p}+r \cos \theta, x_{2}^{p}+r \sin \theta, x_{3}\right)\right| \leq 2 M r
$$


Then, there exists a positive real $M_{3}$ such that, for every $r \geq r_{\varepsilon}, y \in D_{1}, \theta \in[0,2 \pi]$ and $x_{3} \in[0,1]$,

$$
\left|w_{\varepsilon}^{p}\left(x_{3}, y\right)-u\left(x_{1}^{p}+r \cos \theta, x_{2}^{p}+r \sin \theta, x_{3}\right)\right| \leq M_{3} r .
$$

Thus the following estimation for $\partial u_{\varepsilon} / \partial r$ can be derived

$$
\begin{aligned}
\mid \frac{\partial u_{\varepsilon}}{\partial r}( & \left.x_{1}^{p}+r \cos \theta, x_{2}^{p}+r \sin \theta, x_{3}\right) \mid \\
= & \mid(1-\gamma(r)) \frac{\partial u}{\partial r}\left(x_{1}^{p}+r \cos \theta, x_{2}^{p}+r \sin \theta, x_{3}\right) \\
& +\frac{d \gamma}{d r}\left(w_{\varepsilon}^{p}\left(x_{3},(\cos \theta, \sin \theta)-u\left(x_{1}^{p}+r \cos \theta, x_{2}^{p}+r \sin \theta, x_{3}\right)\right) \mid,\right. \\
& \leq M+M_{3}\left|\log \left(\frac{r_{\varepsilon}}{R_{\varepsilon}}\right)\right|^{-1} .
\end{aligned}
$$

Finally, it is easy to verify that $\left|\partial w_{\varepsilon}^{p} / \partial x_{3}\right|$ is bounded; then there exists $M_{4}$ such that

$$
\begin{gathered}
\left|\frac{\partial u_{\varepsilon}}{\partial x_{3}}\left(x_{1}^{p}+r \cos \theta, x_{2}^{p}+r \sin \theta, x_{3}\right)\right| \\
=\mid(1-\gamma(r)) \frac{\partial u}{\partial x_{3}}\left(x_{1}^{p}+r \cos \theta, x_{2}^{p}+r \sin \theta, x_{3}\right) \\
+\gamma(r) \frac{\partial w_{\varepsilon}^{p}}{\partial x_{3}}\left(x_{3},(\cos \theta, \sin \theta)\right) \mid \leq M_{4}
\end{gathered}
$$

The estimations (44), (45), (46) imply that $\left|\nabla u_{\varepsilon}\right|$ is bounded on each layer $C_{\varepsilon}^{p}$, and thence on the set $C_{\varepsilon}$. As $\left|C_{\varepsilon}\right|$ tends to 0 , there follows $\lim \sup \int_{C_{\varepsilon}}\left|\nabla u_{\varepsilon}\right|^{2} d x=0$ and

$$
\lim \sup E^{m}\left(C_{\varepsilon}, u_{\varepsilon}\right)=0 .
$$

Assertion (iii) of Theorem 1 is proved by the estimations (42), (43) and (47).

\section{Comments}

Due to the properties of $\Gamma$-convergence, our result is still valid when external body forces are present. Indeed, a term $\int_{\Omega} f(x) u(x) d x$ can be added to both $E_{\varepsilon}$ and $E_{0}$. In that way, we can solve non-trivial equilibrium problems.

Our result states that the homogenized material is a second gradient material: it has a "three dimensional bending stiffness" $k$. This is not so surprising: it is well know that elastic cylinders, when their radius tends to zero, behave like rods (which are second gradient one-dimensional media): in a sense, we studied the homogenized properties of a system of rods connected by an elastic matrix. However, it must be emphasized that such a result could not be reached by considering directly an elastic matrix reinforced by one-dimensional rods (there is no interaction between a one-dimensional and an elastic three-dimensional medium).

The limit energy $E_{0}$ contains a remaining classic elastic part, $E^{m}(\Omega, u)$. One could consider, afterwards, the limit $\left(\mu_{0}, \lambda_{0}\right) \rightarrow(0,0)$ in $E_{0}$ and obtain an energy depending only on the second gradient of the displacement (the bending stiffness $k$ does not depend on $\mu_{0}$ or $\lambda_{0}$ ).

The particular features of second gradient materials, like the hyperstress tensor [13, 14], flux of interstitial working [12, 10], edge forces [11], presence of a force distribution of order one with respect to the normal derivative [19] can be interpreted in our particular case as limits of some microscopic elastic forces.

An open question raised by our study is the general condition for the change of differential order of the energy when passing to the limit. We already pointed out that such a change was impossible for scalar problems. Our feeling is that the properties of the kernel of the energy density (rigid motions in our case) is essential: it leads to constraints verified by the limit of sequences with bounded energy (in our case these constraints (27) are stated in the proof of Lemma 6). They may be some partial differential equations 
which increase the differential order of the energy. However, they also depend strongly on the geometry: for instance, we do not yet know whether it is possible to find a limit energy depending on a higher gradient of the displacement (third or higher order gradient material) by changing the distribution of the high rigidity inclusions.

Acknowledgements. This paper was initiated by enlightening discussions with M. Bellieud and G. Bouchitté who studied non-local effects for scalar problems in the same geometry $[5,4]$.

\section{References}

1. Acerbi E Buttazzo G Percivale P (1988) Thin inclusions in linear elasticity: a variational approach, J. Reine. Angew. Math. 386, pp $99-113$

2. Allaire G (1992) homogenization and two scale convergence, SIAM J. Math. Anal. 23, 6, pp 1482-1518

3. Barré de Saint-Venant A.J.C (1856) Mémoire sur la flexion des prismes, Journal de Liouville, 2ème série, t.1

4. Bellieud M. and Bouchitté G (1997) Homogénéisation de problèmes elliptiques en présence de fibres de grandes conductivité, C. R. Acad. Sci. Paris., t. 323, série I, pp 1135-1140

5. Bellieud M (1997) Homogénéisation de problèmes elliptiques avec effets non locaux, Thèse de l'Université de Toulon

6. Beurling A Deny J (1959) Dirichlet spaces, Proc. Nat. Acad. Sci. U.S.A. 45, pp 208-215

7. Cailllerie D (1981) Homogénéistation d'un corps élastique renforcé par des fibres minces de grande rigidité et réparties périodiquement, C. R. Acad. Sc. Paris, Série II, t.292, pp.477-480.

8. Cosserat E. and Cosserat F (1909) Sur la théorie des corps déformables, Herman, Paris.

9. Dal Maso (1993) An introduction to $\Gamma$-convergence. Progress in non linear differential equations and their applications, Birkhauser, Boston

10. Dell'Isola F. and Seppecher P (1995) The relationship between edge contact forces and interstitial working allowed by the principle of virtual power, C. R. Acad. Sci. t. 321, série IIb, pp 303-308

11. Dell'Isola F. and Seppecher P (1997) Edge Contact Forces and Quasi-Balanced Power, , Meccanica 32, pp 33-52

12. Dunn J.E (1986) Interstitial working and non classical continuum thermodynamics, in New perspectives in thermodynamics, J. Serrin Ed., Springer Verlag, Berlin, pp 187-222

13. Germain P (1973) La méthode des puissances virtuelles en mécanique des milieux continus. Premiere partie: Théorie du second gradient, Journal de Mécanique, Vol. 12, N. 2, pp 235-274

14. Germain P (1973) The method of virtual power in continuum mechanics. Part 2: Microstructure, S.I.A.M. J. Appl. Math., Vol. 25, N. 3, pp 556-575

15. Le Dret H (1991) Problèmes variationnels dans les multi-domaines, Research Notes in Applied Mathematics, Ciarlet, Lions Ed., Masson, Paris

16. Nguetseng G (1989) A general convergence result for a functional related to the theory of homogenization, SIAM J. Math. Anal. 20, 3, pp 608-623

17. Pideri C. and Seppecher P (1997) Un résultat d'homogénéisation pour un matériau élastique renforcé périodiquement par des fibres élastiques de très grande rigidité, C. R. Acad. Sci. Paris, t. 324, Série II b, pp 475-481

18. Sanchez-Palencia E (1980) Non Homogeneous Media and Vibration Theory, Springer-Verlag

19. Seppecher P (1989) Etude des conditions aux limites en théorie du second gradient: cas de la capillarité, C. R. Acad. Sci. Paris, t. 309, Série II, pp 497-502.

20. Seppecher P (1996) A numerical study of a moving contact line in Cahn-Hilliard theory, Int. J. Engng. Sci. Vol 34., No 9, pp.977-992 